\title{
Bortezomib-based Triplet Regimens for Remission Induction in Multiple Myeloma
}

\author{
Saima Humayun Toor, Tariq Mahmood Satti, Raheel Iftikhar, Syed Kamran Mahmood, Ghassan Umair Shamshad \\ and Jahanzeb Ur Rehman
}

Armed Forces Bone Marrow Transplant Centre / National Institute of Blood and Marrow Transplant, Rawalpindi, Pakistan

\begin{abstract}
Objective: To compare the efficacy and side effect profile of different bortezomib-based triplet regimens for remission induction in patients with multiple myeloma (MM).

Study design: Observational study.

Place and Duration of study: Armed Forces Bone Marrow Transplant Centre, Rawalpindi from January 2014 to December 2018.

Methodology: A total of 81 patients of MM, were registered from January 2014 to December 2018. In final analysis, 44 out of 81 patients were included as per inclusion/exclusion criteria. Bortezomib-based regimens were used either as first line (in newly diagnosed) or as second line (in relapsed/refractory bortezomib naïve patients) therapy. Three different bortezomib-based triplet therapies were used (1) VCd, (2) VRd and (3) VTd. As there were only two patients in VTd regimen group so for study purposes VRd and VTd were grouped together, i.e. Vd with an IMiD. Response to treatment was assessed using the IMWG criteria. Comparison between different bortezomib-based regimens was performed in terms of their tolerability and response rate after four cycles of chemotherapy.

Results: Out of 44 patients, $79.5 \%(n=35)$ patients received bortezomib-based triplet regimen as first line therapy, and in $20.5 \%(n=9)$ patients as second line therapy. VCd was administered to $56.8 \%(n=25)$ and Vd with an IMiD was used in $43.1 \%$ ( $n=19$, VRd in 17 and VTd in 2) of the patients. Response was assessed at the end of fourth cycle. Overall response rate was comparable in both groups, $88 \%$ in VCd versus $89.4 \%$ in Vd with an IMiD group ( $p=0.432$ ). In VCd and Vd with an IMiD group, CR was observed in $52 \%(n=13)$ and $57.9 \%(n=11)$ patients, respectively. Disease remained stable in $6.8 \%(n=3)$ patients. Treatment was generally well tolerated. Comparative analyses of both treatment groups revealed that the frequency of peripheral neuropathy was significantly higher in Vd with an IMiD group (47.3\% vs $8 \% p=0.03)$. Grade III/IV neuropathy observed in $15.7 \%(n=3)$ of the patients in Vd with an IMiD group vs none in VCd group. Grade III/IV cytopenias were more seen in VCd group then in Vd with an IMiD group ( $16 \%$ vs. $5.2 \%$ p=0.16).

Conclusion: The overall response rates were comparable in VCd and Vd with IMiD, with a better side effect profile seen with VCd regimen.
\end{abstract}

Key Words: Multiple myeloma, Bortezomib, Triplet therapy, Remission induction, Peripheral neuropathy

How to cite this article: Toor SH, Satti TM, Iftikhar R, Mahmood SK, Shamshad GU, Rehman JU. Bortezomib-based Triplet Regimens for Remission Induction in Multiple Myeloma. J Coll Physicians Surg Pak 2020; 30(05):527-531. DOI: https://doi.org/10.29271/jcpsp.2020.05.527.

\section{INTRODUCTION}

Multiple myeloma (MM) is a plasma cell dyscrasia that makes up $1 \%$ of all cancers and $10 \%$ of hematologic neoplasms. ${ }^{1}$ It is characterised by malignant proliferation of plasma cells in the bone marrow associated with the abnormal and excessive production of monoclonal immunoglobulin (M protein), which can either be its subclass IgG, IgA, IgD, IgE, and IgM or its light chain kappa and lambda in serum or urine. ${ }^{2}$ Survival outcomes have improved considerably from approximately 3 years to more than 10 years. ${ }^{3}$

Correspondence to: Dr. Saima Humayun Toor, Armed Forces Bone Marrow Transplant Centre / National Institute of Blood and Marrow Transplant, Rawalpindi, Pakistan E-mail:dr.saima.humayun@gmail.com

Received: February 21, 2020; Revised: May 30, 2020;

Accepted: May 30, 2020

DOI: https://doi.org/10.29271/jcpsp.2020.05.527
This is chiefly attributed to advances in understanding of MM pathology leading to provision of improved and novel treatment options. ${ }^{4}$ Several new therapeutic options introduced for the treatment of MM during the past 2 decades include immune-modulatory drugs (IMiD) like thalidomide, lenalidomide, and pomalidomide; and proteasome inhibitors like bortezomib and carfilzomib. In particular, these drugs have been used as part of induction therapy prior to autologous stem cell transplant (ASCT) and as consolidation/maintenance after ASCT. ${ }^{6}$

Currently, 3-drug combinations are the standard of care for remission induction prior to ASCT. These include bortezomib, dexamethasone plus either an IMiD or cyclophosphamide or doxorubicin. ${ }^{\text {In }}$ prospective Phase 2 and Phase 3 clinical trials, the most commonly used triplet regimens were bortezomib-thalidomide-dexamethasone (VTd), bortezomib-lenalidomide-dexamethasone (VRd), and bortezomib-cyclophosphamide-dexamethasone (VCd). These have demonstrated high response rates and better depth of response preceding ASCT and longer progression 
free survival (PFS) ${ }^{8-10}$ When compared head-to-head in phase 2 and 3 trials, the results of these regimens are comparable.,11,12 Immune approaches such as adoptive cellular therapies, vaccines, or antibody-based immune manipulations are the emerging paradigms in the treatment of MM. ${ }^{13}$ Yet, several recommendations of MM treatment cannot be implemented in underdeveloped countries due to financial concerns and availability of novel agents. ${ }^{5}$

To date, no comparative data regarding response to the bortezomib- based triplet regimens for remission induction in $\mathrm{MM}$ patients in Pakistan was available. This study aimed at determining the efficacy and safety of these regimens in patients of MM treated in this centre.

\section{METHODOLOGY}

It was a retrospective analysis of bortezomib-based triplet regimens for remission induction in patients of MM who were treated at Armed Forces Bone Marrow Transplant Center/National Institute Of Blood And Marrow Diseases (AFBMTC/NIBMT), Rawalpindi after approval by the Institutional Review Board.

A total of 81 patients of MM were registered from January 2014 to December 2018. Inclusion criteria were cases of MM who were diagnosed as perIMWG criteria and received treatment with bortezomib-based triplet regimen either as first line therapy, i.e. in newly diagnosed or as second line therapy, i.e. in relapsed/refractory bortezomib naïve cases. Patients who received non-bortezomib-based therapy or bortezomib-based doublets therapy for remission induction were excluded from the study. Patients who were lost to follow-up before completion of treatment or those who received bortezomib-based induction therapy at some other centre and were later referred to AFBMTC/NIBMT for ASCT, were also excluded from the study. Based on these criteria, 44 patients were included in the final analysis. Data variables include age, sex, type of M protein band, ISS staging, induction regimen used, response to therapy, frequency and severity of treatment emergent adverse events (TEAEs).

Three different bortezomib based triplet therapies were used (1) Bortezomib-cyclophosphamide-dexamethasone (VCd), (2) Bortezomib-lenalidomide-dexamethasone (VRd), and (3) Bortezomib-thalidomide-dexamethasone (VTd). Bortezomib was given in a dose of $1.3 \mathrm{mg} / \mathrm{m}^{2}$ and dexamethasone $40 \mathrm{mg}$ both at days 1 , $8,15,22$ of the chemotherapy cycle. The third drug included either cyclophosphamide at $300 \mathrm{mg} / \mathrm{m}^{2}$ on days $1,8,15,22$; or lenalidomide $25 \mathrm{mg}$ at days 1 to 14 (alternatively $10 \mathrm{mg}$ on days 1 to 21 ) or Thalidomide at a dose of $100 \mathrm{mg}$ on days 1 to 21 in 4-week cycles for four cycles. For study purposes VRd and VTd were grouped togetheri.e. Vd with an IMiD.

Response to therapy was assessed after four cycles of chemotherapy according to international myeloma working group (IMWG) criteria as complete remission (CR), very good partial response (VGPR), partial response (PR), stable disease or progressive disease. TEAEs were graded as per National Cancer Institute common terminology criteria for adverse events (CTCAE) v5.0. Comparison between different bortezomib-based triplet regimens was made in terms of their efficacy (response rate) and tolerability.
Data was analysed in SPSS version 24.0. Mean value \pm SD was calculated for numerical variables, examined by independent sample t-test. Frequencies and percentages were calculated for categorical variables. Chi-square test were used to test the proportion of the response rate and TEAEs between the two groups. The p-values less than 0.05 were regarded as significant.

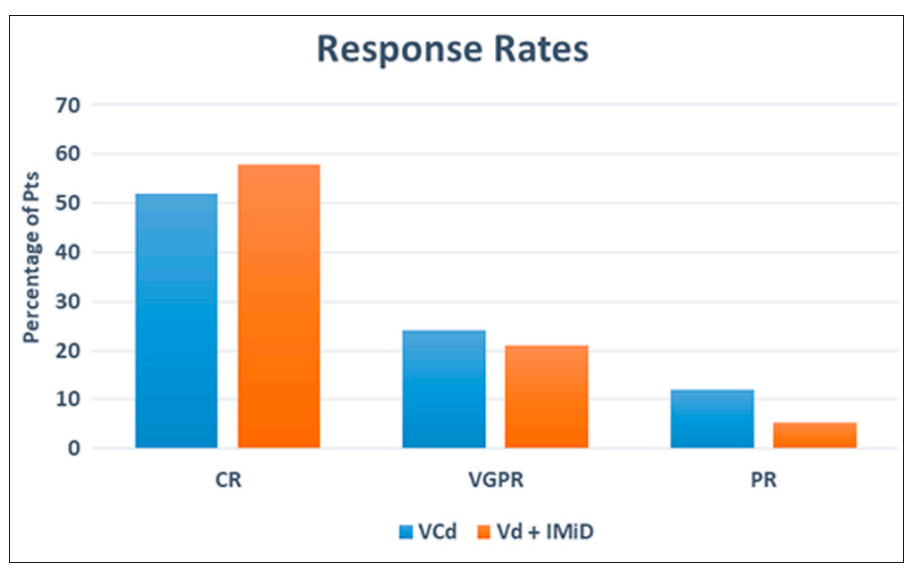

Figure 1: Response rates in bortezomib-based tripletregimens.

VCd:Bortezomib+Cyclophosphamide+dexamethosone.

VdwithIMiD:Bortezomib+dexamethasone+lenalidomide/thalidomide.

CR:Completeremission, VGPR:Verygood partialremission,PR:Partialremission.

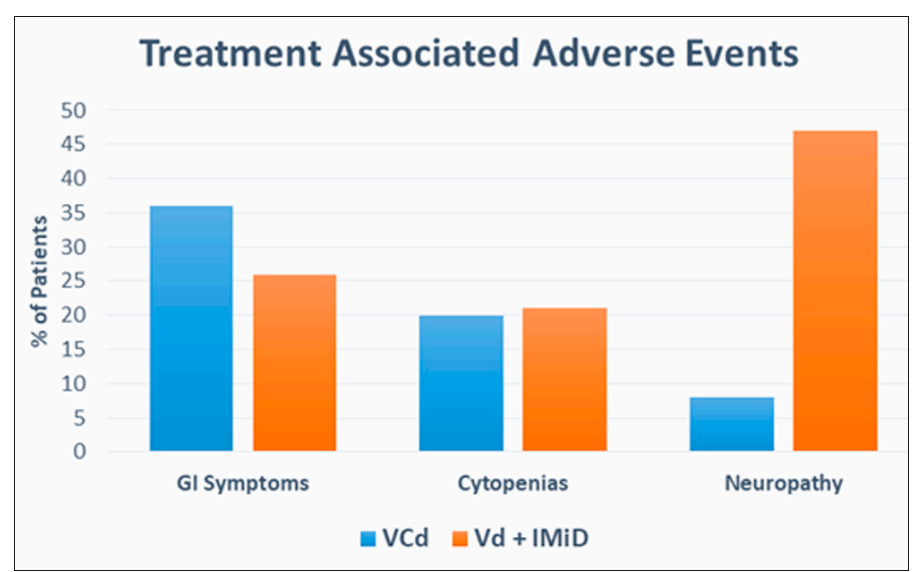

Figure 2: Safety profile of bortezomib-based triplet regimens.

\section{RESULTS}

Among the 44 patients, 29 (66\%) were males and 15 (34\%) were females. The median age of the patients was 56 years with a minimum of 33 years and maximum of 81 years. Median age was 55 years males and 58 years in females.

The most common monoclonal paraprotein identified was IgG kappa, i.e. in $23(52.3 \%)$ patients. This was followed by lgG lambda in $9(20.5 \%)$, IgG kappa with extramedullary plasmacytoma in 5 (11.3\%), IgA kappa in $3(6.8 \%)$, IgA lambda and free light chain only in $2(4.5 \%)$ patients each.

As per international staging system (ISS), Stage I, II and III disease were present in 7 (16\%), 18 (41\%) and 19 (43\%) of the patients. Revised-ISS was not applied as required investigationsi.e. cytogenetics and fluorescent in situ hybridization (FISH) were not performed at time of diagnosis. In VCd group, 4 (16.0\%) patients presented with Stage I disease while stage II and III disease was present in $11(44 \%) \& 10(40 \%)$ patients respectively. In Vd with an IMiD group, Stage I, II and III disease was present in 3 (15.7\%), 7 
(36.8\%) and 9 (47.3\%) patients respectively. The mean value of $B_{2}$ microglobulin in patients with Stage I, II and III disease was 2.54 $\pm 0.28,4.45 \pm 0.21$ and $12.76 \pm 2.09 \mathrm{mg} / \mathrm{dL}$ respectively. Whereas, the mean values of albumin in Stage I, II and III were $4.2 \pm 0.13), 3.6$ \pm 0.16 and $3.5 \pm 0.28 \mathrm{~g} / \mathrm{dL}$ respectively. Table I shows the characteristics of the patients before treatment.

Table I: Baseline characteristics of the patients.

\begin{tabular}{|l|c|c|}
\hline Patient characteristics $(\mathbf{n = 4 4 )}$ & VCd $(\mathbf{n = 2 5 )}$ & Vd+IMiD (n=19) \\
\hline Male / Female & $17 / 8$ & $12 / 7$ \\
\hline Median age & 55 years & 58 years \\
\hline $\begin{array}{l}\beta_{2} \text { microglobulin } \mathrm{mg} / \mathrm{L} \\
\text { (median) }\end{array}$ & $1.2-14.5(7.8)$ & $2.1-13.7(7.9)$ \\
\hline Albumin g/L (median) & $2.2-5.3(3.7)$ & $2.8-7.5(5.1)$ \\
\hline $\begin{array}{l}\text { Disease stage } \\
\text { International staging system) }\end{array}$ & $4(16 \%)$ & $3(16 \%)$ \\
ISS I & $11(44 \%)$ & $7(37 \%)$ \\
ISS II & $10(40 \%)$ & $9(47 \%)$ \\
ISS III & $8.6-13.8(11.2)$ & $8-12.2(10.1)$ \\
\hline Hemoglobin g/dl (median) & $144-342(243)$ & $121-376(248)$ \\
\hline Platelet count x10 $/$ (median) & $3-9.8(6.4)$ & $4-8.5(6.2)$ \\
\hline WBC count x10 ${ }^{9} / L$ (median) & $46-137(114)$ & $52-178(115)$ \\
\hline Creatinine umol/L (median) & & \\
\hline
\end{tabular}

Bortezomib-based triplet regimen, as first line therapy, was given in $79.5 \%(n=35)$ and as second line therapy in $20.5 \%(n=9)$ patients. VCd was administered to $56.8 \%(n=25)$ and $\mathrm{Vd}$ with an IMiD was used in $43.1 \%(n=19)$ of the patients. Out of 19 patients who received Vd with an IMiD group; Ienalidomide (VRd) was used in 17 and thalidomide (VTd) was used in 2 patients. Overall response rate was comparable in both groups, $88 \%(n=22)$ in VCd versus $89.4 \%(n=17)$ in Vd with an IMiD group $(p=0.432)$. In VCd and $\mathrm{Vd}$ with an IMiD group CR was observed in $52 \%(n=13)$ and $57.9 \%(n=11)$ patients respectively. The detail break up of response in both the groups is shown in Figure 1.0. Disease remained stable in $6.8 \%(n=3)$ patients. Out of these three patients, one was from VCd and two were from Vd with an IMiD group.

Bortezomib-based triplet regimen was used as second line therapy in 9 patients. A sub-analysis of these relapse/refractory bortezomib naïve MM patients who received VCd or Vd with an IMiD as a second line therapy showed $55.5 \%(n=5)$ received VCd and $44.5 \%(n=4)$ received Vd with an IMiD (VRd). In VCd group $(n=5), C R, V G P R$ and PR were observed in $20 \%(n=1), 40 \%(n=2)$, $40 \%(n=2)$ patients respectively while in Vd with an IMiD group $(n=4), C R$ and VGPR were observed in 50\% $(n=2)$ and $25 \%(n=1)$ patients respectively and disease remained stable in $25 \%(n=1)$ patient.

Both the triplet regimens were generally well tolerated. Gastrointestinal symptoms, cytopenias and peripheral neuropathy were the commonly observed TEAEs which were graded according to $\mathrm{NCl}$ CTCAE v5.0. In VCd group GI symptoms, cytopenias and peripheral neuropathy were seen in 36\% $(n=9), 20 \%(n=5)$ and $8 \%(n=2)$ patients respectively as compared to $\mathrm{Vd}$ with an IMiD group where GI symptoms, cytopenias and peripheral neuropathy developed in $26.3 \% \quad(n=5), 21 \%(n=4), 47.3 \% \quad(n=9)$ patients respectively. Comparative analysis of both treatment groups revealed that the incidence of peripheral neuropathy was significantly higher in Vd with an IMiD group ( $47.3 \%$ vs $8 \% p=0.03$ ) with grade III/IV neuropathy observed in $15.7 \%(n=3)$ of the patients vs none in VCd group. Grade III/IV cytopenias were more seen in VCd group then in Vd with an IMiD group ( $16 \%$ vs. $5.2 \% \mathrm{p}=$ $0.16)$ but none of these patient developed grade III/IV infection. Safety profile of the two groups shown in Figure 2.

\section{DISCUSSION}

Diagnostic and therapeutic modalities in multiple myeloma are changing rapidly. The shifts that are occurring impact the management of these patients, from initial diagnosis through multiple relapses. The goal of induction treatment is to achieve the highest possible response rate and to avoid adverse events precluding intensive therapy and insufficient collection of stem cells in transplant eligible patients. ${ }^{7}$ Response to induction regimens must be optimized as the quality of response is an important prognostic factor and is predictive of PFS following ASCT.${ }^{14}$ Bortezomib based triplet therapy including dexamethasone and either an IMiD or cyclophosphamide are most commonly administered regimens for transplant eligible as well as transplant ineligible patients. ${ }^{7-10,15}$ The results of the IFM 2005 - 01 Phase III trial which compared for the first time, the efficacy and the safety of a bortezomib-containing induction regimen with conventional chemotherapy including vincristine/doxorubicin/dexamethasone (VAD) and VAD + dexamethasone, cyclophosphamide, etoposide and cisplatin (DCEP) before autologous stem-cell transplantation in multiple myeloma (MM) patients concluded that the novel agent based induction therapy (bortezomib/dexamethasone) achieved higher complete remission (CR)/nearCR rates, as well as less treatment-related mortality. However, it had higher rates of polyneuropathy than the conventional chemotherapy based induction therapy. ${ }^{16}$ The addition of a third agent to $\mathrm{Vd}$ (eg, thalidomide [VTd], doxorubicin [PAD], lenalidomide [RVd], or cyclophosphamide results in improved response rates over the $\mathrm{Vd}$ combination alone. ${ }^{17}$ In a randomised phase 3 PETHEMA/GEM study, the median progression-free survival (PFS) was significantly longer with VTd (56.2 vs. 28.2 vs. 35.5 months, $p=0.01$ ) and the postASCT CR rate was higher with VTd than with Td (46\% vs $24 \%, p=$ 0.004). ${ }^{10}$ The UPFRONT and the PETHEMA trials comparing the efficacy of different bortezomib-based combinations have been unable to establish the superiority of one specific combination. ${ }^{18}$ Currently, different regimens are used interchangeably with different institutional preferences, as well as according to patients baseline characteristics, i.e. co-morbidities, renal functions at the time of diagnosis.

In this observational study, we performed a retrospective head to head com parative analysis of efficacy and safety of different bortezomib-based triplet regimens used for remission induction in patients of MM treated at the study center. Our study is in agreement with a comparative phase 2 trial (EVOLUTION) comparing bortezomib, dexamethasone, cyclophosphamide and lenalidomide with similar overall response rate between $\mathrm{VCd}$ and $\mathrm{Vd}$ with an IMiDs group. ${ }^{9}$ Our results are contrary to those reported by the IFM2013-04 trial whichstrongly confirms the significant and synergistic activity of an IMiD combined with bortezomib and dexamethasone. In IFM2013-04 trial, although CR rate was similar in 2 arms, the VGPR rate (the primary end point) was significantly higher in the VTd $\operatorname{arm}(66.3 \%$ vs $56.2 \% ; p=0.05$; difference $10.1 \%$; 
$95 \% \mathrm{Cl}, 1 \%$ to $18 \%)^{7}{ }^{7}$ While in this study, both CR and VGPR rates were comparable in two groups. In VCd group significantly higher rate of CR i.e. $52 \%$ vs $6 \%$ reported by Cavo et al. ${ }^{19}$ could be attributed not only to increase number of cycles (four vs. three) but also to increase dose of cyclophosphamide in each cycle (four doses of $300 \mathrm{mg} / \mathrm{m}^{2}$ per cycle vs two doses of $500 \mathrm{mg} / \mathrm{m}^{2}$ per cycle) used in our study. In this study, the frequently used IMiDs was Lenalidomide rather than thalidomide and this was in accordance with an integrated analysis of randomized control trial evaluating the VRd or VTd induction in MM. It concluded that VRd had a significantly higher VGPR rate than VTd when 6 cycles of each treatment were compared in transplant eligible newly diagnosed MM patients. This supported the favorable benefit-risk profile with VRd over VTd. ${ }^{12}$ Previous phase II and III comparative analysis of bortezomib based regimen only included newly diagnosed cases of $\mathrm{MM}$, but in this study were included both the newly diagnosed and relapsed/refractory bortezomib naïve patients of MM. Six out of nine patients in RRMM group had received thalidomide with dexamethasone as first line therapy. One patient received lenalidomide with dexamethasone and two received VAD as first line therapy. Overall response rate was similar $88.8 \%$ as compared with those who received bortezomib based regimens as first line therapy thus emphasising the central role of proteasome inhibitors in triplet regimens being used for remission induction in multiple myeloma.

The main limitations of this study were that consolidation and maintenance treatment was not analysed and the data for PFS or OS was not collected.

The safety profile of different regimens used in this study was also interpreted. Overall VCd was better tolerated in these patient population and incidence of adverse events was more in $\mathrm{Vd}$ with an IMiD group. Among the frequently observed side effects, grade III/IV peripheral neuropathy (PN) was more associated with Vd with an IMiD group (15.7\%) than VCd group in which no grade III/IV PN was noted. Pathophysiological and molecular mechanisms underlying PN induced by the concurrent use of these novel agents are mostly unknown. ${ }^{20}$ While maintaining the quality of response rate, one of the reason to switch thalidomide to lenalidomide in triplet regimen is to reduce peripheral neuropathy. ${ }^{7}$ Frequency of PN was similar to that previously described in large phase 3 trials (10\% to $35 \%$ ) in which bortezomib was administrated IV biweekly and not sub-cutaneous. ${ }^{10,20,21}$ Although sub-cutaneous bortezomib was preferable to IV preparation, ${ }^{22}$ in our study only 9 out of total 41 patients and two out of three patients who developed grade III/IV PN received IV bortezomib probably because of non-availability of SC bortezomib at that time. Although a large Italian trial that investigated VTD induction followed by ASCT reported that PN was reversible in the majority of patients, i.e in the VTD group, $88 \%$ of PN completely resolved within 73 days and $94 \%$ improved to at least grade 1 within a median time of 78.5 days, PN can be irreversible and may impair the quality of life of a patient. ${ }^{20}$ Therefore, it is an important issue while selecting the specific induction regimen for $\mathrm{MM}$.

\section{CONCLUSION}

The comparison of different bortezomib-based triplet regimens showed that overall response rates were comparable in VCd and
Vd with IMiD, with a better side effect profile seen with VCd regimen. Selection of remission induction regimen should, therefore, be based on patient's performance status, comorbidities and tolerance to drug side effects.

\section{CONFLICT OF INTEREST:}

Authors declared no conflict of interest.

\section{AUTHORS' CONTRIBUTION:}

TMS: Contribution to the conception or design of the work, or the acquisition, analysis, or interpretation of data for the work. Agreement to be accountable for all aspects of the work in ensuring that questions related to the accuracy or integrity of any part of the work are appropriately investigated and resolved. Final approval of the version to be published.

SKM, RI, JUR: Contribution to the conception or design of the work, or the acquisition, analysis, or interpretation of data for the work. Agreement to be accountable for all aspects of the work in ensuring that questions related to the accuracy or integrity of any part of the work are appropriately investigated and resolved.

GU: Drafted the work or revised it critically for important intellectual content. Agreement to be accountable for all aspects of the work in ensuring that questions related to the accuracy or integrity of any part of the work are appropriately investigated and resolved.

\section{REFERENCES}

1. Palumbo A, Attal M, Roussel M. Shifts in the therapeutic paradigm for patients newly diagnosed with multiple myeloma: Maintenance therapy and overall survival. Clin Cancer Res 2011; 17:1253-63.

2. Zhu W, Chen W. Bortezomib-based treatment for multiple myeloma patients with renal impairment: A systematic review and meta-analysis of observational studies. Medicine 2016; 95:e5202.

3. Landgren O, Iskander K. Modern multiple myeloma therapy: Deep, sustained treatment response and good clinical outcomes. J Intern Med 2017; 281:365-82.

4. Terpos E, Katodritou E, Rubia J, Hungria V, Hulin C, Roussou $M$, et al. Bortezomib-based therapy for relapsed/refractory multiple myeloma in real-world medical practice. Eur J Haematol 2018; 101:556-65.

5. Hameed A, Ali J, Munawar K, Arshad F, Badar F, Siddiqui. Characteristics and outcomes of patients with multiple myeloma: Data from a developing country. Med J Islam Repub Iran 2018; 32:1.

6. Cavo M, Rajkumar SV, Palumbo A, Moreau P, Orlowski R, Blade J, et al. International myeloma working group consensus approach to the treatment of multiple myeloma patients who are candidates for autologous stem cell transplantation. Blood 2011; 117:6063-73.

7. Moreau P, Hulin C, Macro M, Caillot D, Chaleteix C, Roussel M. VTD is superior to VCD prior to intensive therapy in multiple myeloma: results of the prospective IFM2013-04 trial. Blood 2016; 127:2569-74.

8. Richardson PG, Weller E, Lonial S, Jakubowiak AJ, Jagannath S, et al. Lenalidomide, bortezomib, and dexamethasone combination therapy in patients with newly diagnosed multiple myeloma. Blood 2010; 116:679-86.

9. Kumar S, Flinn I, Richardson PG, Hari P, Callander N, Noga SJ, et al. Randomized, multicenter, phase 2 study (EVOLUTION) 
of combinations of bortezomib, dexamethasone, cyclophosphamide, and lenalidomide in previously untreated multiple myeloma. Blood 2012; 119:4375-82.

10. Rosiñol L, Oriol A, Teruel All, Hernández D, López-Jiméne J, de la Rubia J, et al. Superiority of bortezomib, thalidomide, and dexamethasone (VTD) as induction pretransplantation therapy in multiple myeloma: A randomized phase 3 PETHEMA/GEM study. Blood 2012; 120:1589-96.

11. Jimenez-Zepeda V, Bahlis NJ, Duggan P, Alonso R, Lahuerta J, Valeri A, et al. Cyclophosphamide, bortezomib and dexamethasone (CyBorD) compared to bortezomib, thalidomide and dexamethasone (vtd) as induction therapy for the treatment of transplant eligible multiple myeloma. Blood 2016; 128:4505.

12. Rosinol Dachs L, Hebraud B, Orio A, Colin A, Rios R, Hulin C, et al. Integrated analysis of randomized controlled trials evaluating bortezomib + lenalidomide + dexamethasone or bortezomib + thalidomide + dexamethasone induction in transplant-eligible newly diagnosed multiple myeloma. Blood 2018; 132:3245.

13. Hari P. Recent advances in understanding multiple myeloma Hematol Oncol Stem Cell Ther 2017; 10:267-71.

14. Moreau P, Attal M, Facon T. Frontline therapy of multiple myeloma. Blood 2015; 125:3076-84.

15. Usmani SZ, Hungria VT, Leleu X, Lee HC, Davies FE, Costello $C$, et al. Transplant status does not impact the selection of induction regimens for newly diagnosed multiple myeloma (ndmm) patients (pts) in the insight $\mathrm{mm}$ prospective, observational study. Blood 2018; 132:3289.

16. Harousseau JL, Attal M, Avet-Loiseau H, Marit G, Caillot
$\mathrm{D}$, Mohty $\mathrm{M}$, et al. Bortezomib plus dexamethasone is superior to vincristine plus doxorubicin plus dexamethasone as induction treatment prior to autologous stem-cell transplantation in newly diagnosed multiple myeloma: results of the IFM 2005 - 01 Phase III trial. J Clin Oncol 2010; 28: 4621-9.

17. Moreau $\mathrm{P}$, Attal M, Facon T. Frontline therapy of multiple myeloma. Blood 2015; 125:3076-84.

18. Kapoor P, Ramkrishan V, Rajkumar SV. Bortezomib combination therapy in multiple myeloma. Semin Hematol 2012; 49:228-42.

19. Cavo M, Pantani L, Pezzi A, Petrucci MT, Patriarca F, Di Raimondo $\mathrm{F}$, et al. Bortezomib-thalidomide-dexamethasone (VTD) is superior to bortezomib-cyclophosphamidedexamethasone (VCD) as induction therapy prior to autologous stem cell transplantation in multiple myeloma. Leukemia 2015; 29:2429-31.

20. Tacchetti P, Terragna C, Galli M, Zamagni E, Petrucci MT, Pezzi A, et al. Bortezomib- and thalidomide-induced peripheral neuropathy in multiple myeloma: Clinical and molecular analyses of a phase 3 study. Am J Hematol 2014; 89:1085-91.

21. Moreau $\mathrm{P}$, Avet-Loiseau $\mathrm{H}$, Facon T, Attal M, Tiab M, Hulin $C$, et al. Bortezomib plus dexamethasone versus reduceddose bortezomib, thalidomide plus dexamethasone as induction treatment before autologous stem cell transplantation in newly diagnosed multiple myeloma. Blood 2011; 118:5752-8

22. Mu SD, Ai LS, Qin Y, Hu Y. Subcutaneous versus intravenous bortezomib administration for multiple myeloma patients: $\mathrm{A}$ meta-analysis. Curr Med Sci 2018; 38:43-50. 